vulnerable to reverse causation and confounding. Mendelian randomization (MR) studies with even large numbers of unrelated individuals can suffer from familial biases, and dynastic effects ('genetic nurture') can be especially pronounced for socially patterned phenotypes like obesity. We introduce a 'within-family' MR (WFMR) design that uses genotyped mother-father-offspring trios to overcome these biases.

Methods In 5237 8-year-old children from the Norwegian Mother, Father and Child Cohort Study (MoBa), we estimated the effects of body mass index (BMI) on symptoms of depression, attention-deficit hyperactivity disorder (ADHD), anxiety, and autism spectrum disorder (ASD). Child height, weight, and outcomes were based on mother-reported information from questionnaires. We used polygenic risk scores (PRS) as instrumental variables for BMI, with and without adjustment for parents' own PRS. PRS were calculated in PRCise, and all other analysis completed in STATAv15. PRS were calculated using genetic variants from the largest and most recent genome-wide association study (GWAS) for BMI.

Results Initial MR estimates implied a one-unit higher BMI increased depression symptoms by 0.12 (95\%CI $0.05,0.20)$ and ADHD symptoms by $0.11(0.03,0.18)$ standard-deviations, and reduced symptoms of anxiety by $-0.11(-0.19$,$0.03)$ and symptoms of ASD by $-0.05(-0.13,0.03)$. For ASD, associations did not differ substantially between symptoms related to social communication $(-0.03(-0.12,0.05)$ and to repetitive behaviour $(-0.04(-0.12,0.03)$. Accounting for parental genotypes in WFMR made little difference to estimates, with no strong evidence of indirect effects of parental genotypes on offspring phenotype. Next steps will be to examine relationships using gene variants associated with BMI during childhood specifically, and to investigate the influence of depressive, ADHD, anxiety and ASD symptoms on childhood BMI.

Conclusion Influence of childhood BMI on emotional and neurodevelopmental health is not explained by family-level genetic biases, suggesting childhood BMI may affect these symptoms. Negative associations of BMI with anxiety are consistent with results from the UK Biobank, where genetic propensity for BMI was negatively associated with risk of selfreporting as a 'nervous' person. Intervening on childhood BMI may influence these outcomes.

\section{OP97 EFFECTS OF AN ALCOHOL PREVENTION PROGRAM AT SPORT ARENAS: A 2-YEAR QUASI-EXPERIMENTAL FOLLOW-UP STUDY USING PSEUDOPATRONS AND BREATH ALCOHOL CONCENTRATION LEVELS}

${ }^{1,2} \mathrm{TH}$ Elgan*, 1,2 N Durbeej, ${ }^{1,2} \mathrm{~J}$ Gripenberg. ${ }^{1}$ Clinical Neuroscience, Karolinska Institute, Stockholm, Sweden; ${ }^{2}$ STAD, Centre for Psychiatry Research, Stockholm, Sweden

\subsection{6/jech-2020-SSMabstracts.95}

Background Alcohol intoxication and related problems among spectators are common at large sporting events around the world. In Sweden, the problems are greatest in the Swedish Premier Football League (SPFL). According to the Alcohol Act, obviously intoxicated individuals are not allowed to enter or purchase alcohol at arenas. In 2015, we initiated a research project with the aim to reduce intoxication levels among spectators. A multi-component prevention program was co-created and implemented together with key stakeholders, and consisted of community mobilization, training, policy work, and enforcement. The aim of this study was to evaluate the effects of this program.

Methods This is a quasi-experimental control group study using a repeated cross-sectional design (baseline 2015, follow-ups 2016 and 2017). The setting was arenas hosting SPFL games in Stockholm (intervention area) and Gothenburg (comparison area). Professional actors (i.e. pseudopatrons) were trained to act a standardized scene of obvious intoxication while attempting to enter arenas and purchase alcohol at licensed premises inside arenas. To control for possible external factors, we also conducted alcohol purchase attempts at licensed premises outside arenas, where denial rates should remain stable. Spectators at arenas were randomly invited to provide BrAC-assessments using breathalyzers.

Results A total of 10178 BrAC-assessments were collected, 201 attempts to enter arenas, and 495 attempts to purchase alcohol at premises inside arenas. In the intervention area, denial rates toward obviously intoxicated spectators increased between the assessment points from $12.9 \%$ (95\%CI 6.6-22.2) to $32.8 \%(22.5-44.6)$ at entrances and from $31.8 \% 25.4$ $39.0)$ to $56.8 \%(49.6-63.7)$ at premises inside arenas. Mean BrAC-level decreased between baseline and two-year follow-up from $0.063 \%(0.061-0.065)$ to $0.057 \%(0.054-0.059)$ and the proportion of spectators with high intoxication levels from $9.7 \%(8.7-10.7)$ to $5.9 \%(5.1-6.9)$. A similar pattern was observed in the comparison area, however, while the denial rate remained stable at premises outside arenas in the intervention area, it increased in the comparison area.

Conclusion Interpretation of results is complex due to improvements in the comparison area. However, unlike in the intervention area, denial rates increased at premises outside the arena in the comparison area, indicating that external factors have affected the arena in the comparison area. Results then suggest that the intervention was successful in increasing staff intervention toward obviously intoxicated spectators, thereby decreasing the overall intoxication levels. A limitation is the repeated cross-sectional design which limits conclusions on causality. The intervention has the potential to be implemented at other sporting events.

\section{OP98 USING DELPHI METHODS TO INCREASE SEASONAL INFLUENZA VACCINATION IN HEALTHCARE WORKERS ACROSS LONDON}

${ }^{1} \mathrm{C}$ Heffernan*, 'S Baracaia, ${ }^{2} \mathrm{~K}$ Aziz. ${ }^{1}$ PHE London Region, Public Health England, London, UK; ${ }^{2}$ Screening and Immunisation, NHS England and Improvement (London), London, UK

\subsection{6/jech-2020-SSMabstracts.96}

Background Seasonal influenza vaccination (SIV) uptake in healthcare workers (HCWs) across London has more than doubled since $2014 / 15$ from $25 \%$ to $64 \%$ in $2018 / 19$, but uptake is still less than the national average of $70 \%$. Given the lack of certainty in the literature around interventions that work to improve uptake, particularly within the NHS and across a range of Trust types, the aim here is to determine which interventions have been effective (and ineffective) in increasing uptake in SIV in HCWs across the 36 acute, community, mental health and specialist trusts in London.

Methods The Delphi technique is a structured process that uses a series of questionnaires or 'rounds' to gather information until a group consensus is reached. An advantage of the methodology is that it can be used to collate expertise across 